\title{
AUTOMATED VISUAL INSPECTION OF ROAD SURFACE CRACKS
}

\author{
Roberto Medina \\ CARTIF Foundation, Boecillo (Valladolid), Spain \\ robmed@cartif.es \\ Jaime Gómez-García-Bermejo \\ University of Valladolid, Valladolid, Spain \\ jaigom@eis.uva.es \\ Eduardo Zalama \\ University of Valladolid, Valladolid, Spain \\ ezalama@cartif.es
}

\begin{abstract}
Pavement maintenance requires knowing the state of the road surface. Human inspection is the most common method for evaluating this state. Recently, the automated visual inspection has been addressed, but some important questions remain open concerning the variable ambient lighting, shadows, device synchronisation and the large amount of data. In the present paper, an automated visual inspection system is presented. Images are obtained using laser lighting and linear cameras onboard a vehicle. Longitudinal and transversal cracks are detected and classified using a novel approach based on combining traditional features and Gabor filters. A Differential Global Positioning System (DGPS), a web camera and an Inertial Profiler to measure the International Roughness Index (IRI) are also considered in order to obtain comprehensive information about the road state. Implementation details are given concerning image acquisition and processing, system architecture and data synchronisation. Field results are presented which prove the suitability of the approach.
\end{abstract}

KEYWORDS: Image processing, pavement inspection, feature extraction, Gabor filters

\section{INTRODUCTION}

Road clearly plays a predominant rule in passengers and goods transport. In 2006, passenger cars, powered two-wheelers and buses and coaches accounted for $83 \%$ of total passenger transport in the twenty-seven member state European Union (EU-27), as it was reported by the European Commission (2009). Also, 46\% of total goods transport is carried out by road. This huge load of vehicles using the road daily, the weather and the environmental conditions cause road deterioration and subsequent loss of properties of the pavement. This causes a number of serious drawbacks for users: reduced road safety, decreased travel comfort, 
increased fuel consumption consequently increased polluting gases emission. Therefore, getting an adequate level of service of the road network becomes a priority.

Pavement maintenance requires the knowledge of the state of the road. Human visual inspection of the road surface is the most common method for evaluating this state, but it does not cope with the required deterioration evaluation reliability, repeatability, speed and robustness. The human visual system has a great ability to adapt to new and variable tasks. However, the same state of the road may be classified in a different way by two different workers, due to the inherent human subjectivity and visual fatigue. Some semiautomated survey systems, working in post-processing mode and requiring substantial human interaction, have also been used. (Wang, 2000). However, in most cases a manual labour is still required for collecting crack data.

In this context, INO's Laser Road Imaging System (LRIS) emerged as an efficient system to acquire quality images of the road. Indeed, many researchers have paid attention to automated pavement distress detection using image analysis and machine vision techniques. Kim et al. (2006) evaluated the crack sealing performance by image processing. Also, Chang et al. (2007) developed an autonomous robot for crack inspection. Zhaoyun et al., (2009) proposed an algorithm to identify pavement cracks upon morphological method.

However, variable external lighting conditions and shadows prevent common thresholding techniques from working successfully, and efficient crack detection remains an open challenge. In the present paper, an approach for the detection of cracks using Gabor filters is proposed. A group of these filters can distinguish between different kinds of image groups, but the significance of filter parameters is unclear (Bianconi and Fernandez, 2007).

Moreover, pavement inspection is not simply a matter of acquiring images and designing image processing algorithms. Information about the pavement state could be enriched by the integration of other devices. A DGPS is used to provide accurate position of detected cracks. A web camera is employed to provide information of the location. An Inertial Profiler is used to measure the IRI. Details of these devices and its synchronisation using an encoder will be also discussed.

The rest of the paper is organised as follows. First, the system architecture is presented, addressing the synchronising problem. Then, the algorithms proposed for improving the image quality and detecting and classifying cracks upon Gabor filters are explained. The results and conclusions are finally given.

\section{SYSTEM ARCHITECTURE}

A wide variety of devices are set up in the vehicle in order to acquire the required information. An imaging system is used for acquiring road images. An Inertial Profiler is used to measure IRI, derived from longitudinal profiles of pavement. These longitudinal profiles and the IRI give extra information of the road state. A web camera and a DGPS provide accurate position of the vehicle and images of the road, respectively. Two computers are used for controlling the device, storing data and processing this data offline for obtaining information about the road state. Finally, all these devices are synchronized using an encoder attached to a wheel. Setup details are seen in Figure 1. 


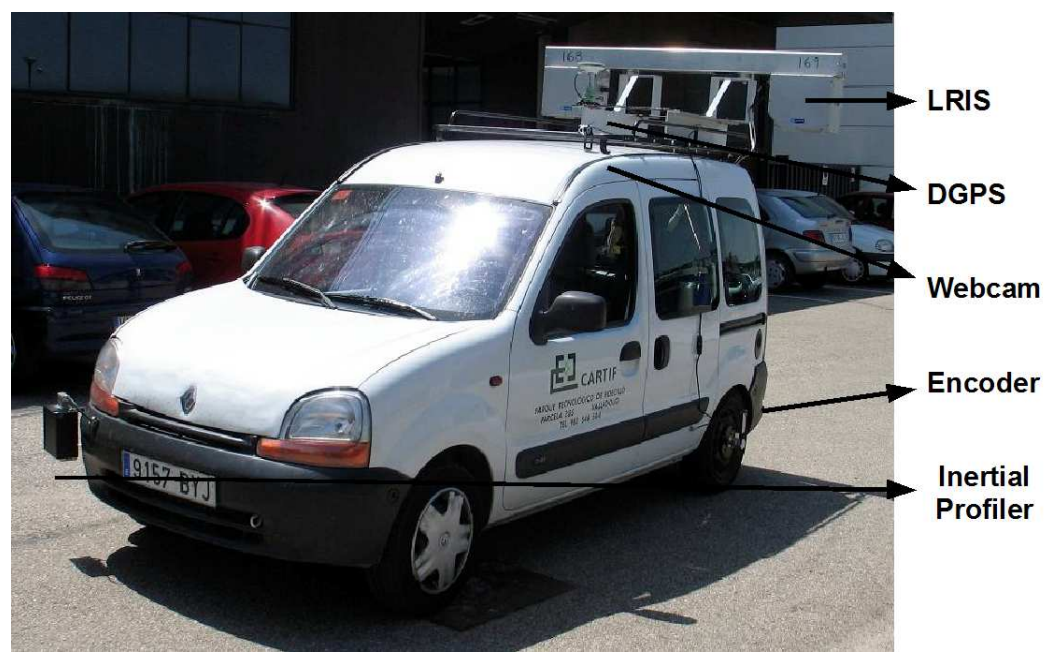

Figure 1: Road Inspection Vehicle. LRIS, a DGPS, a webcam, an encoder and an inertial profiler have been integrated into the vehicle.

In the present section, first, the encoder and the way the rest of the devices are synchronised are explained. Then data acquisition devices are described.

\section{Devices synchronisation using an encoder}

An incremental encoder is used to synchronise the different devices of the system. It gives 5,000 pulses each wheel turn, which means 1 pulse every time the vehicle advances $0,33 \mathrm{~mm}$.

Every device must start and stop the data acquisition at the same time. This is achieved by starting all the system devices without powering the encoder. When all the systems are ready to start acquiring data, then the encoder is powered on, thus providing the first encoder pulse at the same time to all devices. Analogously, acquisition is stopped by powering off the encoder, so all devices receive the last pulse at the same time.

Each device must take a single data every a given number of encoder pulses. The inertial profiler uses the normal encoder signal, but it takes data at the laser frequency, so it uses the encoder signal to position the data acquired. LRIS acquires a transverse line image of the road every time a pulse is received. A frequency divisor has been used in order to generate a pulse every 3 pulses of the encoder, so that one line every $1 \mathrm{~mm}$ is acquired. The DGPS and the web camera give a data every 5000 pulses. An I/O card allows pulse counting and sends a signal to the software every 5000 pulses. When this signal is received, the web camera takes an image and the position given by the DGPS is saved. A proper interface software has been also developed, that allows the user to analyse all the stored data from the devices.

\section{Imaging system}

In order proper images of the road surface to be acquired, the cameras should provide high enough data acquisition and transfer speed so that the incoming light integrated during the short exposition times is enough to produce significant output values. The use of matrix cameras could be considered. However, linear cameras are preferred because their acquisition 
speed is higher and uniform and high-power lighting is more easily achieved along a line than throughout a 2D area. Suitable lighting is also required to obtain high quality images. Most usual lighting sources are halogen lamp, Light Emitting Diode (LED) and laser. Halogen lamps require high energy so they are not fully appropriate for on-board systems. LEDs have better power efficiency than halogen lamps, but worse efficiency than lasers. Moreover, laser lighting requires high quality manufacturing alignment with the linear cameras.

Based on these considerations, semiautomated imaging systems have been developed and are available on the market. Automatic Road Analyzer (ARAN), developed by from FrugoRoadware, uses digital frame cameras and synchronised strobe lighting and stores the images for post-processing by the WiseCrax software. The Highways Agency Road Research Information System (HARRIS) applied three line scan cameras and halogen lighting. The pixel resolution is about $2 \mathrm{~mm}$ and can operate at up to $80 \mathrm{~km} / \mathrm{h}$ with a covering width $2.9 \mathrm{~m}$. Automatic processing of these images is then carried out in two stages. First, a processing step to clean and reduce the images and then the identification of cracks is carried out.

INO's LRIS consists of two high-resolution linear cameras and two lasers to image $4 \mathrm{~m}$ transverse road sections. The line size obtained in LRIS is 4096 pixels and 28000 lines every second, which allows $1 \mathrm{~mm}$ resolution at speeds that can reach $100 \mathrm{~km} / \mathrm{h}$. The system is designed to increase the contrast and visibility of both longitudinal and transversal road cracks. Using high power laser line projectors with a wavelength of $825 \mathrm{~nm}$, it can operate in full daylight because it is immune to variations in outside lighting conditions and shadows. The incident illumination angle causes the cracks to project shadows, so the visual appearance of the cracks is usually darker than the normal road surface. LRIS achieves our requirements of acquisition speed, resolution image and power consumption. In the present work, this system has been selected and integrated in the vehicle. Images are processed using specific software we have developed upon the methods proposed in the next section.

\section{Inertial Profiler}

The International Roughness Index (IRI), derived from longitudinal profiles of pavement, is measured by an Inertial Profiler. The selected equipment is the LaserProf from Greenwood Engineering. LaserProf has a laser, an inertial sensor, an encoder coupled to the wheel, and a control system to integrate all the data. It runs at $16 \mathrm{kHz}$ update frequency, so that a longitudinal profile resampling interval of $1.39 \mathrm{~mm}$ is achieved at $80 \mathrm{~km} / \mathrm{h}$.

The longitudinal profile offers extra information about the state of the road. The IRI is the roughness index determined by applying a mathematical model, which has the dynamic response of a simulated response-type road roughness measuring vehicle along a single wheel-path of measured road profile. The IRI is expressed in terms of accumulated vertical displacement of the simulated suspension in metres per measured kilometre. This information has been incorporated to our system to increase the information about the state of the road.

\section{Differential Global Positioning System}

A DGPS gives an accuracy measurement of the position of the vehicle. This measurement is taken every 1,7 meters and is triggered by the encoder. So the system gives the exactly location of every crack. 


\section{Web camera}

A web camera has also been installed in the vehicle in order to obtain a video of the road. Each frame of the video is taken every 1.7 meters, so it is independent from the speed of the vehicle. The encoder also triggers the acquisition of each frame.

\section{PROPOSED INSPECTION PROCESS}

The design of an automated visual inspection system must consider three basic steps: preprocessing, segmentation and classification. The proposed approaches for these steps are presented in this section. First, a preprocessing step to correct the measured brightness levels along the images is presented. Segmentation algorithms are then used to separate crack regions from the rest of the image. A novel method to analysed images is presented. It is based on applying a segmentation algorithm to filtered images by Gabor filters. Crack regions are then classified into two groups: transverse cracks and longitudinal cracks.

\section{Correction of measured brightness levels}

At the beginning of the process, the gain and the exposure time of every camera is adjusted individually to a 128 average grey level (the centre of the 0 to 255 range). Nevertheless, brightness measured along a given line is not constant due to the fact that the lighting and viewing conditions are not exactly the same at every point. In order to correct existing differences, the following process has been developed. Every time a new image is transferred, the average pixel value for each column is recalculated to adapt to the sheet reflection changes. This is carried out using:

$$
\begin{aligned}
& I_{i j}^{\prime}=128 \frac{I_{i j}}{A_{i}^{k}} \quad i=1,2, \ldots, M \quad j=1,2, \ldots, N \\
& A_{i}^{k}=\alpha A_{i}^{k-1}+(1-\alpha) a_{i}{ }_{i}^{k} \quad i=1,2, \ldots, M \\
& \text { where } \quad \alpha=\beta \frac{k-1}{k}
\end{aligned}
$$

$I_{i j}$ is the pixel value of the original image at column $i$ and the row $j$, and $I_{i j}$ is the transformed image pixel value. $M$ is the number of columns of the image, $N$ is the number of rows of the image and $k$ is the number of the image. Moreover, $A_{i}{ }^{k}$ is the weighted average pixel value of column $i$ after $k$ images and $a_{i}{ }^{k}$ is the average pixel value of column $i$ for image $k . \alpha$ is a weighting variable and $\beta$ is a constant to control the weight of the nearest measurements, which can be set to about 0.75. $\alpha$ approaches $\beta$ when $k$ approaches infinite, in our case 0.75 . An image acquired by LRIS and the corresponding preprocessed image by the algorithm developed can be seen in Figure 2. 


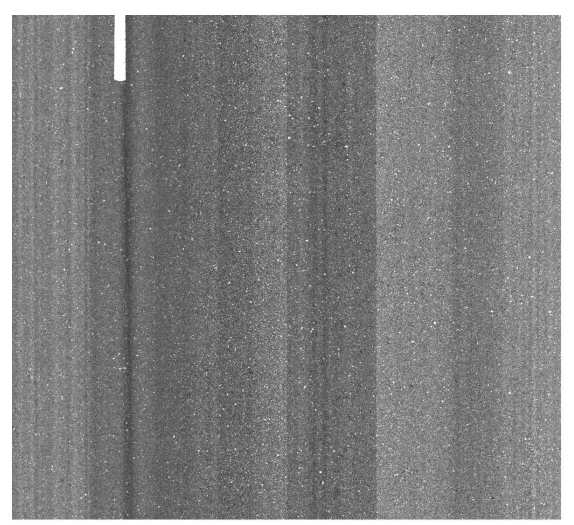

(a)

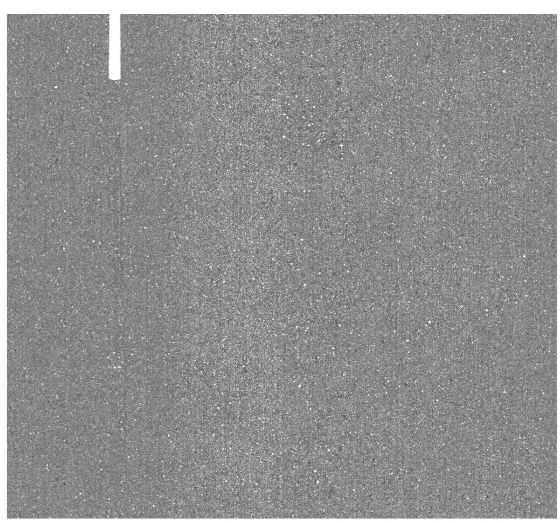

(b)

Figure 2: (a) Image acquired by LRIS. (b) Preprocessed image.

\section{Segmentation of filtered images by Gabor filters}

In the segmentation phase the pixels of an image are grouped. Usually, when detecting defects over a homogeneous surface is looked for, the image is segmented into two groups: normal surface (background) and defective surface (foreground). A natural way to segment an image is through thresholding techniques. All pixels with grey level values greater than a given one are assigned to one group and the rest to another one. Most of these techniques indirectly use the shape information of an image histogram. The ideal case is a bimodal shape, because the grey level at the valley can be directly selected as a suitable threshold value. However, usually bimodal histograms are not found in real applications.

Measured grey level of cracks is usually darker than normal surface, but the differences are not as great as would be desirable. Some samples about thresholding application on images from LRIS system, where this issue is highlighted, can be seen in figure 4. So, a more reliable, robust and less sensitive to noise and intensity variation method should be used.

Many works suggest that analysing images in the spatial-frequency domain instead of the spatial domain can be advantageous. Fourier methods characterise the spatial-frequency distribution but do not consider the information in the spatial domain. Gabor filters are well recognised as a joint spatial/spatial-frequency representation for analysing images containing specific frequency and orientation characteristics, (Bovik et al. 1992).

A two-dimensional Gabor filter consists of a sinusoidal wave modulated by a Gaussian envelope. The filter performs a localised and oriented frequency analysis of a twodimensional signal. The spatial domain formulation is

$$
G_{\sigma, F, \vartheta}(x, y)=g_{\sigma}(x, y) \cdot \exp \left(j 2 \pi F x^{\prime}\right)
$$

where $g_{\sigma}(x, y)=\frac{1}{2 \pi \sigma_{x} \sigma_{y}} \exp \left(-\frac{1}{2}\left(\left(\frac{x^{\prime}}{\sigma_{x}}\right)^{2}+\left(\frac{y^{\prime}}{\sigma_{y}}\right)^{2}\right)\right)$

with $x^{\prime}=x \cos \theta+y \sin \theta$ and $y^{\prime}=-x \sin \theta+y \cos \theta$. 
$x, y$ are the spatial coordinates, $F$ is the central frequency, $\theta$ is the angle between the direction of the sinusoidal wave and the x-axis, and $\sigma_{x}$ and $\sigma_{y}$ are the smoothing parameters (standard deviations of the Gaussian envelope in the direction of the wave and orthogonal to it).

A group of filters selected in such a way that they can distinguish between different kinds of image groups is called Gabor filter bank. The design of the filter bank consists of selecting a proper set of values for the filter parameters: $F, \theta, \sigma_{x}$ and $\sigma_{y}$. The systematic design of a proper Gabor filter bank has been widely studied, but the solution always depends on the specific application.

Once the bank of filters is selected the images are filtered. To compute the filtered images, the full image is divided into fixed size pieces $(512 \times 512$ for the developed system) with the necessary overlapping to cover the entire image. The convolution theorem is applied to these pieces in the following way:

$$
I^{\prime}(x, y)=I(x, y) * G(x, y)=\mathfrak{I}^{-1}(I(u, v) G(u, v))
$$

Where $I$ represents the image, $G$ the filter, $I^{\prime}$ the filtered image and $F^{-1}$ is the inverse Fourier transform. It should be noted that $I^{\prime}$ is a complex image where the real part, imaginary part, magnitude and phase can all be used to segment the image.

Two filters have been used in this work. The central frequency and the smoothing parameters are the same for both of them, while the orientation is different. The central frequency, $F$, has been set up to 0,025 pixels $^{-1}$, the smoothing parameter $\sigma_{x}$ has been set up to 9,64 pixels and $\sigma_{y}$ has been set up to 37,68 pixels. $\theta$ has been set up to 0 degrees and 90 degrees respectively.

A transverse and a longitudinal crack and the filtered images by the two Gabor filters used can be seen in Figure 3. Real and imaginary part, magnitude and phase of the filtered images are shown. It should be notice that the filtered images are normalised in order to see them, representing in black the smaller values. In fact, the values of the pixels of real and imaginary part are real numbers. (i.e., (g) pixel values are between -20.85 and +9.5 ), the magnitude values are positive values and the phase values are in degrees from -180 to +180 .

Real part and magnitude seems to be the most suitable for our purpose. An appropriate threshold has to be selected in order to segment an image. In Figure 4 the segmentation result for two transverse and two longitudinal crack can be seen, applied to the real part and the magnitude, and is also compared to a common thresholding output. The pixels selected in the thresholding technique are those ones whose values are lower than 85. It can be seen that a lot of noise is present in the segmented image. Filtering techniques should be applied to remove the noise, which could cause the elimination of the crack. Moreover, for some cracks, like in (f), it is not possible to detect this kind of cracks using this technique. When a thresholding technique is applied to the real part or to the magnitude, the cracks are finely detected and the noise is almost removed. For the real part two threshold values, upper and lower, are applied. It has been chosen -10 and +10 respectively. An upper threshold of 10 has been selected for the magnitude. There is no relevant difference in the results for both options. Since the magnitude phase only uses one limit, it results in a more homogeneous and robust result. So, the magnitude of the filtered images is finally used. 


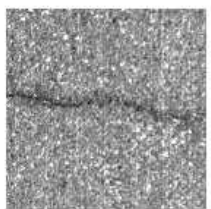

(a) Transverse crack

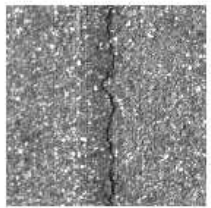

(f) Longitudinal crack

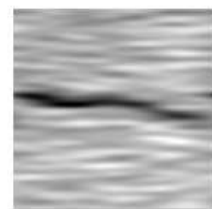

(b) Real part

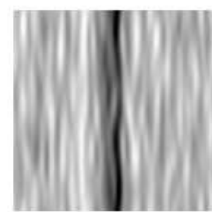

(g) Real part

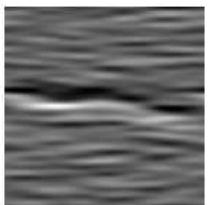

(c) Imaginary part

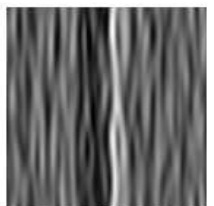

(h) Imaginary part

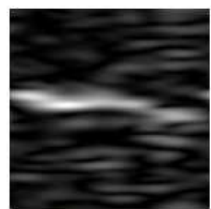

(d) Magnitude

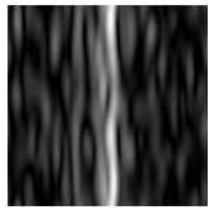

(i) Magnitude

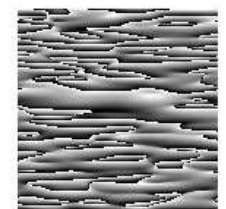

(e) Phase

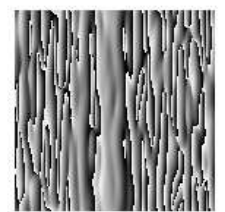

(j) Phase

Figure 3: (a) and (f) are two cracks images acquired by LRIS system. (b) and (g) are the real part of the filtered images by two Gabor filters, (c) and (h) are the imaginary part, (d) and (i) the magnitude, and $(e)$ and $(j)$ the phase.

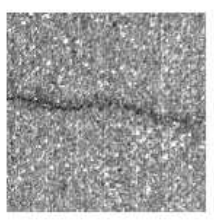

(a) Transverse cadk

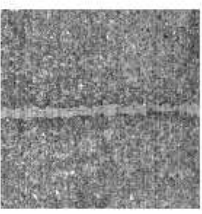

(e) Transverse dadk

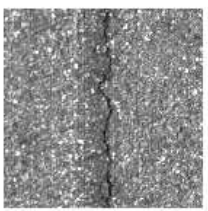

(i) Longitudinal crack

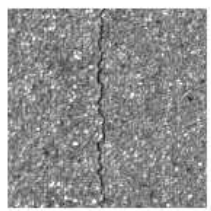

(m) Longitudinal crack

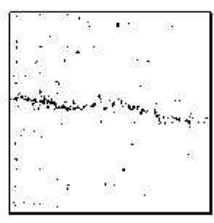

(b) Typical Thresholding

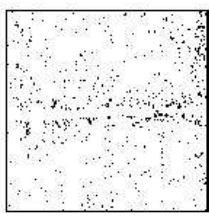

(f) Typical Thresholding

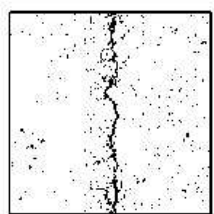

(a) Typical Thresholding

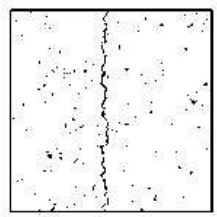

(n) Typical Thresholding

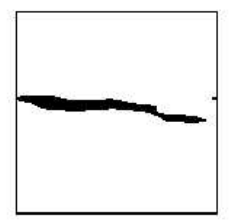

(c) Real part

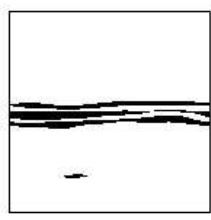

(g) Real part

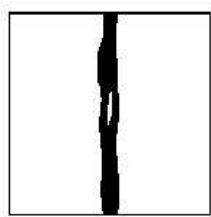

(k) Real part

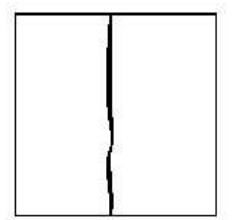

(o) Real part

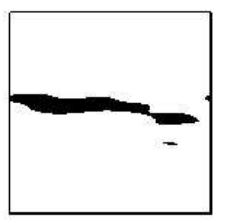

(d) Magnitude

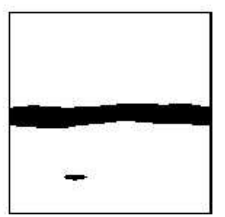

(h) Magnitude

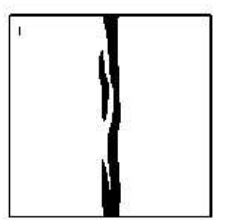

(1) Magnitude

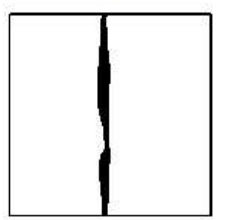

(p) Magnitude

Figure 4: (a) and (e) are transverse cracks, (i) and (m) are longitudinal cracks. (b), (f), (j) and (h) are the corresponding thresholded images by a typical technique. (c), $(\mathrm{g}),(\mathrm{k})$ and $(\mathrm{o})$ is the thresholded real part filtered image and (d), (h), (l) and $(p)$ for the magnitude.

\section{Crack classification}

The pavement cracks are classified into transverse and longitudinal cracks. The segmentation technique, described in the previous section, provides two binary output images. Transverse 
cracks are highlighted in one of them, and longitudinal cracks in the other one. Each of these images is analysed independently. Adjacent pixels corresponding to cracks form groups and nearby groups are joined to form a single defect. This processing allows the number of cracks to be counted up, and the features of these cracks to be calculated. This process is applied to both binary images independently, so the cracks detected in every binary image belong to transverse o longitudinal cracks respectively.

\section{RESULTS}

Once the image data is processed we obtain a detailed map of the state of the road. Classified cracks are shown and located. Information about total amount of cracks, its type, and the environment where the crack is located is also shown. IRI measurement of any road section can be obtained. It could be possible to see the total information acquired by the system, but the amount of data stored is too high. Using the processed data drastically reduces the storage requirements while preserving all the required information. Some screenshots of the interface software developed in this work can be seen in Figure 5.
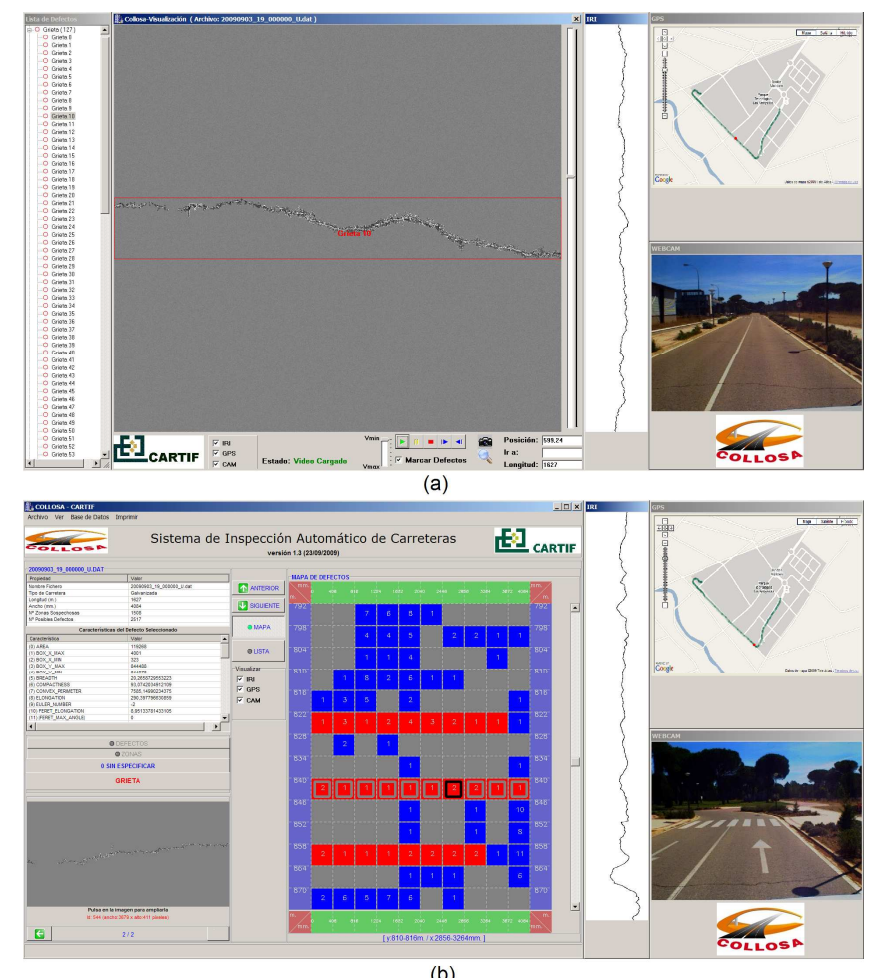

Figure 5: Developed interface to display data: (a) Processed image data, (b) Map of defects, its features and state of the road.

\section{CONCLUSIONS}

In the present paper a new system for detecting and classifying cracks in the road is exposed. It is based on the application of two Gabor filters for the entire image. The results obtained by the application of traditional thresholding techniques have been improved. 
Different devices and its synchronisation have been also explained. These devices give additional information of the state of the road, useful for pavement maintenance.

\section{ACKNOWLEDGMENT}

This work has been partly supported by Contrucciones y Obras Llorente S.A. (COLLOSA), and the Spanish Ministry of Science and Innovation (project PID-560410-2009-12 and PPT020000-2008-6).

\section{REFERENCES}

European Commission (2009) Panorama of Transport - Statistical books. Luxembourg: Office for Official Publications of the European Communities

Wang, K.C.P. (2000) Design and Implementation of Automated Systems for Pavement Surface Distress Survey. ASCE Journal of Infrastructure Systems, 6(1), 24-32.

Kim, H., Soleymani, H., Han, S. H., and Nam, H. (2006) Evaluation of asphalt pavement crack sealing performance using image processing technique. International Symposium on Automation and Robotics in Construction (ISARC2006), 341-345.

Chang, J. R., Tseng, Y. H., Kang, S. C., Tseng, C. H., and Wu P.H. (2007) The study in using an autonomous robot for pavement inspection. $24^{\text {th }}$ International Symposium on Automation \& Robotics in Construction (ISARC), 229-234.

Zhaoyun, S., Chaofan, W., and Aimin, S. (2009) Study of image-based pavement cracking measurement techniques. $9^{\text {th }}$ International Conference on Measurement \& Instruments (ICEMI), 2, 140-143.

Bianconi, F. and Fernández, A. (2007) Evaluation of the effects of Gabor filter parameters on texture classification. Pattern Recognition, 40(12), 3325-3335.

Bovik, A.C., Gopal, N., Emmoth, T., and Restrepo, A. (1992) Localized measurement of emergent image frequencies by Gabor wavelets. IEEE Transactions of Information Theory, $38(2), 691-712$. 